\title{
Lichen Planus flare following COVID-19 vaccination: a case report
}

\author{
Astrid Herzum ${ }^{1}$, Martina Burlando ${ }^{2}$, Mattia Molle ${ }^{1}$, Claudia Micalizzi ${ }^{1}$, and A. Parodi ${ }^{1}$ \\ ${ }^{1}$ IRCCS Ospedale Policlinico San Martino \\ ${ }^{2}$ University of Genoa
}

September 25, 2021

\begin{abstract}
We report the third case of lichen planus (LP) following COVID-19 BNT162b2 vaccination in a 59-year-old woman with previous LP. The reactivation of LP in patients with dormant LP, suggests possible vaccine-induced immune dysregulation. We suggest that the already described vaccine-induced up-regulation of Th1 response, may play a relevant role.
\end{abstract}

\section{INTRODUCTION:}

To contain the COVID-19 pandemics, vaccines have been developed and are being administered worldwide. The SARS-CoV-2 m-RNA vaccine Comirnaty (BNT162b2, BioNTech/Pfizer) is a gene-therapy-based vaccine, whose long-term effects are inevitably unknown and need therefore to be intensively studied. ${ }^{1,2}$

BNT162b2 vaccine has been described to up-regulate Th1-cell response, incrementing inflammatory cytokines involved in the pathogenesis of autoimmune diseases, including connective tissue diseases, psoriasis, vitiligo and lichen planus (LP). ${ }^{1-3}$

Currently only two cases of LP following COVID-19 vaccination, and one lichenoid drug eruption (LDE), have been described. ${ }^{3-5}$

\section{CASE PRESENTATION:}

We report a case of LP following BNT162b2 vaccination in a 59-year-old woman with a past history of LP. Two weeks after the second dose of vaccine she presented for newly appeared pruritic skin lesions. She reported no mucosal nor nail lesions, recent infections, habit changes, nor other possibl triggers.

Six years earlier she presented similar, more generalized, papules, clinically and histologically diagnosed as LP, and effectively treated with topical corticosteroids. Lesions had never recurred.

At physical examination, polygonal, purpuric, scaly and slightly elevated papules were seen on the medial side of both ankles and feet (Fig. 1). Close-up examination and dermatoscopy evidenced fine scales on an erythematous background and Wickham's striae (Fig. 2).

Biopsy confirmed the diagnosis of LP and lesions resolved after three weeks of topical high-potency corticosteroids, with relief of the patient.

\section{DISCUSSION:}

This is the third case of LP following COVID-19 vaccination. In line with literature cases, the patient developing LP recrudescence after COVID-19 vaccination was a woman in her late fifties and was successfully treated only with topical therapy, as the first reported literature case. ${ }^{3,4}$ 
Of note, LP has already been associated also to COVID-19 infection, other viral infections and anti-viral vaccinations. HBV vaccination is the most frequently associated to LP and the first reported one, in 1990, followed by influenza and herpes zoster vaccines. ${ }^{3,6-8}$

LP and LDEs occur uncommonly after anti-viral vaccinations and, as in LP cases after COVID-vaccine, mainly affect middle-aged women, reflecting increased risk of autoimmunity in adult females. ${ }^{8}$

The pathogenesis of LP triggered by anti-viral vaccines is not fully understood. Activated auto-cytotoxic CD8 T-lymphocytes induce basal keratinocytes' death, increase of inflammatory cytokines, as IL-5 and IFN- $\gamma$, and finally LP. ${ }^{8}$

Regarding the specific case of COVID-19 vaccine, only three associated LP cases, comprising ours, have been reported up to date. The association must be therefore considered as possibly just casual.

However, the present case and the first literature case, evidenced LP reactivation in patients with dormant LP, possibly triggered by the vaccine. This supports the thesis that vaccine-induced immune dysregulation may reactivate LP, if not completely induce it.

Indeed, it has been reported that the BNT162b2 vaccine induces upregulation of Th1 response which increases the levels of IL-2, IFN- $\gamma$ and TNF $\alpha$, commonly known for being inflammatory cytokines directly involved in the pathogenesis of LP. ${ }^{1,9}$ However, the possible mechanism underlying LP (re)activation after PfizerBiotNtech COVID-19 vaccination is still widely unknown and needs to be further investigated.

In conclusion, it is important to stress that LP lesions, reported in association with COVID-19 vaccination, are benign, successfully cured with topical therapy and do not represent by any means a reason not to undergo COVID-19 vaccination. Nonetheless, dermatologist should be aware of this and all other possible cutaneous reactions associated to COVID-19 vaccines, to promptly recognize and, if needed, treat them, minimizing the patients' discomfort and thereby encouraging the population to undergo vaccination. ${ }^{10}$ Lastly, we highlight the importance of reporting adverse reactions, in order to promote vaccine safety through pharmacovigilance systems.

\section{A cknowledgement:}

The authors have no acknowledgements to declare.

\section{REFERENCES:}

Sahin U, Muik A, Derhovanessian E, et al. COVID-19

vaccine BNT162b1 elicits human antibody and $\mathrm{T}_{\mathrm{H}} 1 \mathrm{~T}$ cell responses [published correction appears in Nature. 2021 Feb;590(7844):E17]. Nature . 2020;586:594-599.

1. Kaur I, Sharma A, Jakhar D, et al. Coronavirus disease (COVID-19): An updated review based on current knowledge and existing literature for dermatologists. Dermatol Ther. 2020;33:e13677.

2. Hiltun I, Sarriugarte J, Martínez-de-Espronceda I, et al. Lichen planus arising after COVID-19 vaccination. J Eur Acad Dermatol Venereol . 2021;35:e414-e415.

3. Merhy R, Sarkis AS, Kaikati J, et al. New-onset cutaneous lichen planus triggered by COVID-19 vaccination [published online ahead of print, 2021 Jul 8]. J Eur Acad Dermatol Venereol . 2021

4. Burlando M, Russo R, Cozzani E, et al. COVID-19 "second wave" and vaccines: the dermatologists' perspective. Int J Dermatol. 2021;60:889-890.

5. Cozzani E, Herzum A, Burlando M, e al. Cutaneous manifestations of HAV, HBV, HCV. Ital J Dermatol Venerol . 2021;156:5-12.

6. Rebora A, Rongioletti F, Drago F, et al. Lichen planus as a side effect of HBV vaccination. Dermatology. 1999;198(1):1-2. doi: 10.1159/000018054. Erratum in: Dermatology 1999;198:222.

7. Lai YC, Yew YW. Lichen planus and lichenoid drug eruption after vaccination. Cutis. 2017;100:E6-E20.

8. Georgescu SR, Mitran CI, Mitran MI, et al. Oxidative Stress in Cutaneous Lichen Planus-A Narrative Review. J Clin Med. 2021;10:2692. 
9. Guljaš S, Bosnić Z, Salha T, et al. Lack of Informations about COVID-19 Vaccine: From Implications to Intervention for Supporting Public Health Communications in COVID-19 Pandemic. Int $J$ Environ Res Public Health . 2021;18:6141.

\section{FIGURE LEGENDS:}

Fig. 1 : Erythematous to violaceous, scaly, polygonal papules of the right foot.

Fig. 2 : Close up of lichen planus papules showing typical Wickham's striae and scales on an erythematous background.

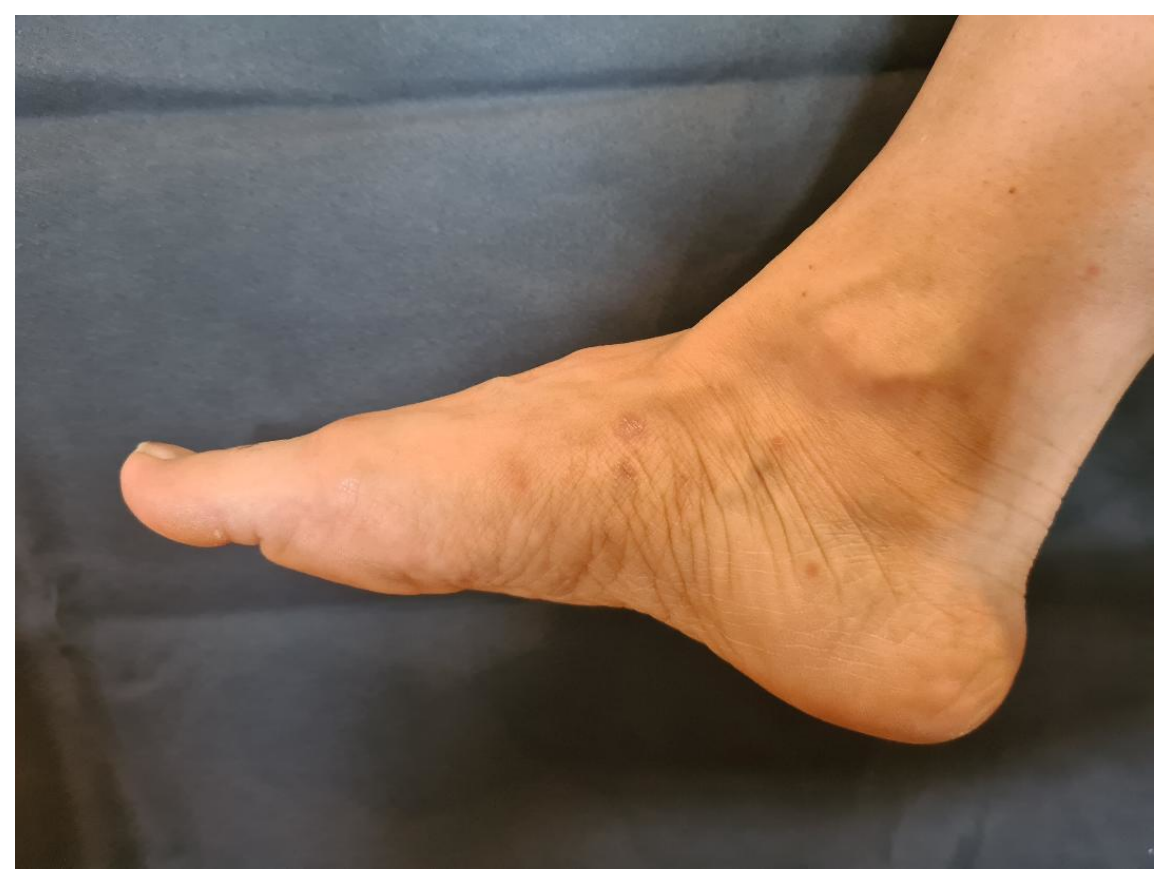




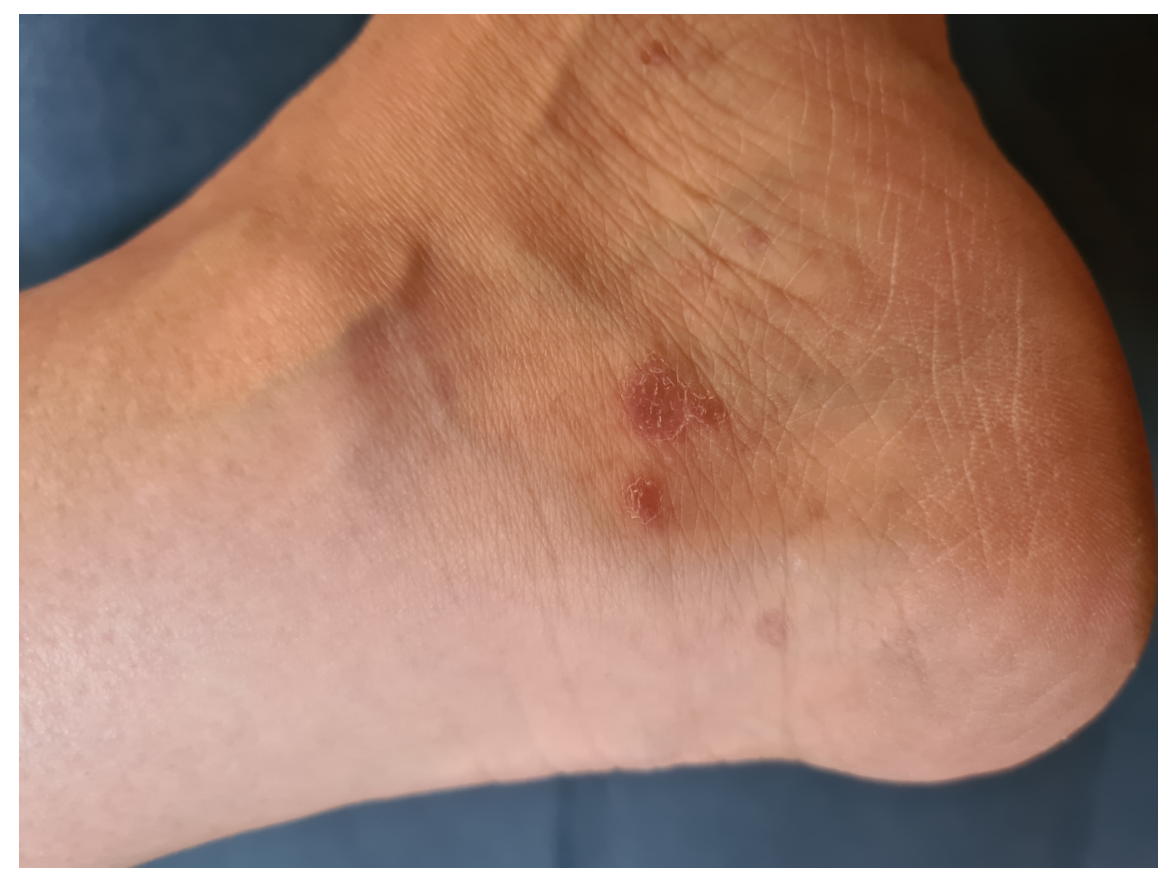

